\title{
Clutter and rainfall discrimination by means of doppler-polarimetric measurements and vertical reflectivity profile analysis
}

\author{
F. Silvestro ${ }^{1}$, N. Rebora ${ }^{1,2}$, L. Ferraris ${ }^{1,2}$, M. Morando ${ }^{1}$, P. Alberoni ${ }^{3}$, and A. Fornasiero ${ }^{1,3}$ \\ ${ }^{1}$ CIMA, Università di Genova e della Basilicata, Savona, Italy \\ ${ }^{2}$ DIST, Università di Genova, Genova, Italy \\ ${ }^{3}$ A.R.P.A Emilia-Romagna - Servizio Idrometeorologico, Italy
}

Received: 24 October 2004 - Revised: 10 February 2005 - Accepted: 16 February 2005 - Published: 9 May 2005

\begin{abstract}
The estimation of rainfall rate and other parameters from radar scattering volume is heavily affected by the presence of intense sea and ground clutter and echoes which appears in anomalous propagation condition. To deal with these non meteorological echoes we present a new clutter removal algorithm which combines the results of previous works. The algorithm fully exploits both the Doppler and polarimetric capabilities of the radar used and the analysis of vertical reflectivity profile in order to achieve the better identification of the meteorological and non-meteorological targets. The algorithm has been applied to the C-band radar of Monte Settepani (Savona, Italy), which runs in a hightopography environment. Preliminary results are presented.
\end{abstract}

\section{Introduction}

One of the primary problems in radar meteorology is to distinguish between rain echoes and those due to ground clutter, artefacts or other types of meteorological targets.

Mountains, building and ground returns can contribute to the received signal, and can cause a corrupted estimate of the precipitation.

A particular case of ground clutter is caused by the socalled anomalous propagation of a transmitted radar beam. Under normal atmospheric conditions, the refractive index of the atmosphere decreases with height above the ground and the radar beam is bent downward and follows more closely the earth surface. In special climatic conditions the radar beam can be bent downward more strongly than normal, so the beam could hit different ground areas against normal conditions.

Another case of echoes due to ground is the second-trip echo; the transmitted signal obviously continues its path also further the range up to which measurements can be per-

Correspondence to: F. Silvestro

(francesco.s@cima.unige.it) formed unambiguously, and can be reflected by targets like ground or mountain. This return can be detected by the radar receiver and interpreted as due to a (fake?) rain target in the radar covered area.

Not only the ground or other fixed objects are the reasons of a bad estimate of rain field. A number of different types of precipitation can exist in atmosphere, such as rain, snow, hail, mixed phase precipitation. The melting layer, where melting ice particles and a water-ice mixture are present, is characterized by high values of reflectivity that do not correspond to high rain rate on the ground.

The aim of this work is to obtain more reliable data that are not (or less) affected by clutter, anomalous propagation and mixed phase hydrometeors.

We based our procedure on two previous works: the first one is about the use of vertical reflectivity profile for identification of anomalous propagation (Alberoni et al., 2001), in the other one the authors find out a method for discriminating rainfall and clutter by means of polarimetric measurements (Giuli et al., 1991).

In this work we propose an algorithm that merges and exploits the advantages of both methods.

The rest of the paper proceeds as follows. In Sect. 2 we describe the method. In Sect. 3 we show some case studies applied to Monte Settepani C-POL radar measurements. Summary and conclusions are drawn in Sect. 4.

\section{The method}

The algorithm consists in a decision tree that combines single tests which will be described in the following paragraphs.

The procedure does not distinguish between unreliable datum and clutter like other methods (e.g. Giuli et al, 1991), our purpose is to decide whether a datum should be used directly for the rain field estimate or it needs to be corrected (or suppressed). 


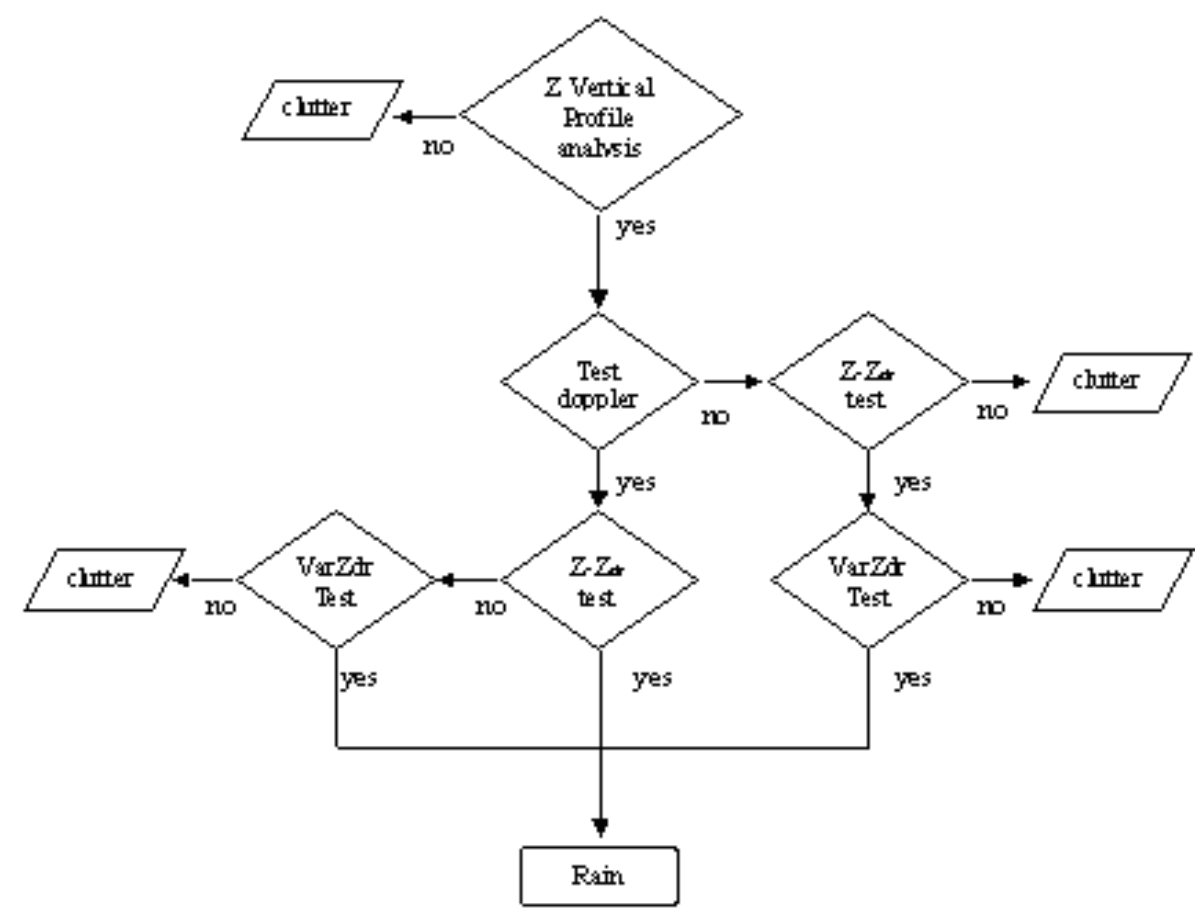

Fig. 1. Flow chart algorithm representation.

\subsection{Doppler test}

The Doppler test identifies those cells with a low magnitude of average Doppler velocity. The cells with velocity lower than a fixed threshold does not pass the test.

The value chosen as threshold is $1.5 \mathrm{~m} / \mathrm{s}$.

Is it clear that the result of this test is not conclusive for detecting the presence of a rain or a clutter echo (Koistinen, 1996).

\section{2 $Z$ and $Z_{D R}$ range test}

This test finds those cells which have $Z_{d r}$ or $Z_{h}$ atypical values.

The $Z_{d r}$ is defined:

$Z_{d r}=10 \log \left(\frac{Z_{H}}{Z_{V}}\right)$

Water drops take the form of nearly oblate spheroids as they fall trough the atmosphere, their degree of eccentricity increases with drop size. Positive values of $Z_{d r}$ are expected in rain phenomena (Ulbrich and Atlas, 1984), moreover because drops with large size are unusual, eccentricity is limited and values of $Z_{d r}$ are not larger then an upper bound. In this work also negative values are considered, these can occurs due to the differential attenuation and to the dispersion of the measured average values in a finite sampling.

The following range is used for $\mathrm{Z}_{d r}:-0.5<Z_{d r}<5 \mathrm{~dB}$ (Vivekanandan et al., 1990).

We must denote that this analysis may be affected by the path attenuation due to the presence of rain.
The limited size of drops and their concentration excludes very large values of $Z_{H}$. The value $60 \mathrm{~dB}$ has been considered as upper bound (Giuli et al., 1991).

\section{3 $Z_{d r}$ spatial variability test}

This test permits to individuate the data windows characterized by a high spatial variability of $Z_{d r}$. A check is performed in windows of 3 azimuth cells by 3 range cells of size. The standard deviation of $\mathrm{Zdr}$ in each window must be lower than a certain threshold, in this work we chose the value $1 \mathrm{~dB}$ (Giuli et al., 1991).

\subsection{Vertical reflectivity profile analysis}

The reflectivity decreases slowly with height if the radar is detecting targets with homogeneous phase, so the vertical reflectivity profile should be smooth. Strong discontinuity can be due to the presence of mountain or anomalous propagation especially for rays of lowest elevations, or to the presence of melting layer.

In rainy situations vertical reflectivity profile should be regular ad smooth.

The technique to investigate the vertical reflectivity profile used in this work has been developed by the Servizio Meteorologico Regionale of the Regione Emilia Romagna in Italy (Alberoni et al., 2001).

The analysis is carried out calculating the difference of reflectivity between two antenna elevation angles:

$\Delta Z=Z_{1}-Z_{2}$

the test classifies the cell as clutter echo if the following conditions are encountered: 
Table 1. Threshold values used in the vertical profile analysis. For the first range bin encountered the "normal" thresholds are used. For other bins of the same beam the "behind thresholds" are used.

\begin{tabular}{ccc}
\hline Threshold (dBZ) & Normal & Behind \\
\hline$T_{1}$ & 30 & 15 \\
$T_{2}$ & -10 & 0 \\
\hline
\end{tabular}

$\Delta Z>T_{1}$ or $\Delta Z>0$ and simultaneously $Z_{2}<T_{2}$

$T_{1}$ and $T_{2}$ are empirical thresholds.

Since the tails of the distributions of $\Delta Z$ for rain echo and for clutter echo overlap a further analysis of data is necessary. An approximate way to solve this problem is establish lower values of thresholds if the previous bin is classified as clutter echo.

The values of thresholds are the same of those used by Alberoni et al. (2001).

\subsection{The complete algorithm}

All the tests briefly described in the previous paragraph have been combined in the decision tree shown in Fig. 1.

The algorithm general principle is that a cell must pass three tests to be classified as rain echo. As shown in the decision tree, the reflectivity vertical profile analysis is more important than the other tests. We did this choice since some non-meteorological echoes show the same characteristics of a rain echo.

For example the second-trip echoes have frequently values of $Z, Z_{d r}$ and $V_{r}$ equivalent to the meteorological ones and despite they are often returns of fixed targets they do not have low values of Doppler velocity. Furthermore also $Z_{d r}$ has not a great spatial variability and its standard deviation is lower than 1 .

In this situation the only way to distinguish if we are observing a non-rain echo is the analysis of the reflectivity vertical profile. Have to be said that also using this kind of investigation not always we manage to detect all the nonmeteorological echoes. This depends on the particular atmospheric conditions during the radar scan, which affect the anomalous propagation and characterizes the quantity and the quality of the non-meteorological echoes.

\subsection{Data correction}

The application of the procedure allows for deciding whether or not a cell should be considered rain echo. The cells classified as clutter echoes are corrected with a very simple procedure: we investigate the data positioned on the upright of the cell that needs to be corrected, when a cell considered rain echo is found, the moments values of this cell are associated to the underneath cells. We recognize that this is a very crude approximation, the reason for this is that we are looking for an operationally oriented technique that could be improved in the future with more sophisticated algorithms using VPR methods.

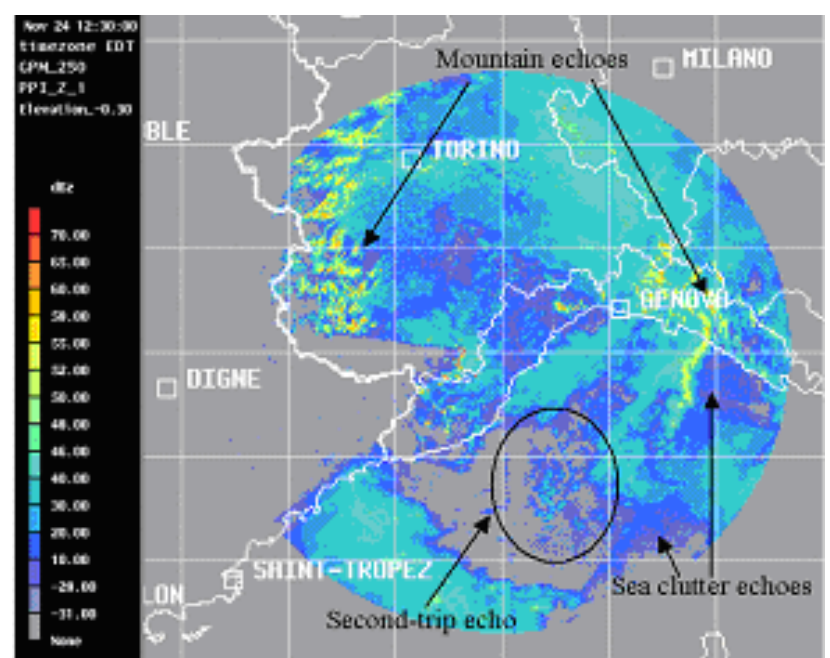

Fig. 2. Reflectivity PPI (dBZ) at $-0.3^{\circ}$ elevation recorded on 24 November 2003 12:30 UTC without filtering.

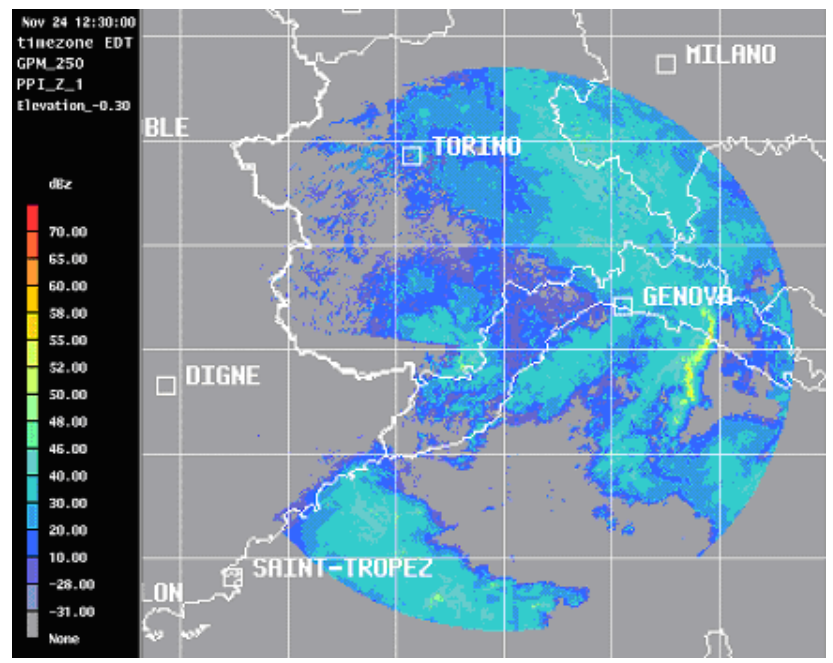

Fig. 3. Reflectivity PPI (dBZ) at $-0.3^{\circ}$ elevation recorded on 24 November 2003 12:30 UTC after the procedure application.

\section{Case studies}

The algorithm has been applied to the C-band polarimetric radar of Monte Settepani (Savona,Italy), which runs in a high topography environment. In the following is shown the result obtained during a rainfall event. The case is an event occurred on the afternoon of 24 November 2003. The data haven't been previously filtered with Doppler filter.

West and East of the radar clutter echoes due to the mountains appear to be embedded in rain echoes (Fig. 2). South of the radar there is a second-trip echoes area, due to the presence of the Corsica island in Mediterranean sea, and sea clutter echoes.

West of the radar there is an area occluded by the presence of high mountains near the radar. When we apply our procedure, as can be seen in Fig. 3, we are able to remove the greater part of clutter echoes and also the second-trip echoes. 


\section{Conclusions}

An algorithm that discriminate between rainfall echoes and clutter echoes has been presented. To reach this task a combination of tests which exploits the proprieties of dualpolarization Doppler measurement and the characteristics of vertical profile of reflectivity has been used. The procedure has been applied in some case of studies and will be implemented in real time applications rain fields estimates.

The preliminary studies have produced good results, though the scheme needs some calibration and adjustments. The analysis of the behavior for different types of precipitation and in different weather conditions will permit to establish if the thresholds used in the combined tests should be changed. Furthermore the update of the static mask used in the vertical reflectivity profile analysis could lead to an improvement of the results.

Acknowledgements. This work is partially supported by CARPE DIEM, a research project supported by the European Commission under the 5th FP (Contract No. EVG1-CT-2001-0045), by RISK AWARE (an INTERREG IIIB - CADSES project), by the GNDCI through the project RAM and by the Regione Liguria. We acknowledge Regione Liguria and Regione Piemonte for putting at our disposal the data of the Radar of Monte Settepani (Savona, Italy).

Edited by: G. Boni

Reviewed by: anonymous referees

\section{References}

Alberoni, P. P., Andersson, T., Mezzasalma, P., Michelson, D., and Nanni, S.: Use of vertical reflectivity profile for identification of anomalous propagation, Meteorol. Appl., 8, 257-266, 2001.

Giuli, D., Gheraradelli, M., Freni, A., Seliga, T. A., and Aydin, K.: Rainfall and clutter discrimination by means of dual-linear polarization radar measurements, J. Atmos.Oceanic Technol., 8, 777-789, 1991.

Koistinen, J.: Clutter cancellation and the capabilities of modern Doppler radar, Proceeding, COST-75 Workshop of weather radar, 7-11, 1996.

Ulbrich, C. W. and Atlas, D.: Assessment of the contribution of differential polarization to improve rainfall measurements, Radio Sci., 19, 49-57, 1984.

Vivekanandan, J., Bringi, V. N., and Raghavan, R.: Multiparameter radar modelling and observations of melting ice, J. Atmos. Sci., 47, 549-564, 1990. 